where even normally reared rats will explore rather than build a nest. When rats deprived in this way are tested in their home cage, they build perfectly good nests. This point has been reiterated in almost all recent animal behaviour textbooks.

Just as serious as what is left out of this book are some of the things which are put into it. There are a number of generalizations which are based on so little fact that they amount to virtual falsehoods. For example, it is stated on p. 219: "The structure of a school is generally regular, with approximately the same number of fishes in each of its three dimensions". In fact, we know very little about the structure of fish schools in any species. And it is misleading to enumerate the functions of play in young animals as if they had been demonstrated with certainty (p. 225).

The basis of the distinction between "higher" and "lower" animals, which is made throughout the book, is not explained (the octopus is described as a "lower invertebrate") and the title of Part 2, "Functional Behaviour Patterns", carries the somewhat curious implication that some behaviour patterns are nonfunctional.

These criticisms may sound unnecessarily severe. They have been expressed strongly because there are now so many books about animal behaviour that the publication of yet another seems justified only if it meets a high standard and has some particular contribution to make. On both these counts this book is unfortunately somewhat lacking.

\section{Marian Dawkins}

\section{Measure for Measures}

Hausdorff Measures. By C. A. Rogers. Pp. viii + 179. (Cambridge University: London, December 1970.) £3.80: \$12.50.

THE book is divided into three chapters, and the titles are respectively "Measure in Abstract, Topological and Metric Spaces", "Hausdorff Measures" and "Applications of Hausdorff Measures".

The first chapter is on general measure theory and contains the fundamentals of the subject. It would be suitable for somebody learning measure theory for the first time. Although the treatment is abstract it is not unduly general. There is considerable emphasis on measures in metric spaces, for this theory is needed in the second chapter.

The second chapter is undoubtedly the chief one. Although the material follows quite logically from the first chapter, the non-specialist might find it difficult. Nevertheless, it is very readable, and a particularly useful feature is that outlines of proofs of theorems are sometimes given before the details. Much of the existing literature of the subject is collected together, and some of the topics are generalized and developed further. Most of the chapter is concerned with general metric spaces, but the case of Euclidean space is briefly discussed in relation to Lebesgue measure and surface areas. One of the principal topics concerns existence theorems. For example, various conditions on the space are proved sufficient to ensure that a compact set of infinite Hausdorff measure contains a compact set of positive finite measure. In this connexion there is a reference to a joint paper by Roy $O$. Davies and the author in which an example is given to show that some restrictions on the space are needed. It seems a pity that this interesting example is not included in the book.

The third chapter is concerned chiefly with Euclidean space and is therefore less abstract. The title of the chapter is somewhat misleading as many of the topics are not so much applications of Hausdorff measure as part of geometric measure theory studied in its own right. Most of these topics are only briefly surveyed. The survey is reasonably complete but there are some omissions. The two topics considered in detail are continued fractions and continuous increasing functions.

This is not just another book on measure theory and it is unique in the ground covered. It is remarkably selfcontained and there is an excellent bibliography. It should be particularly useful to the beginning research mathematician who is interested in measure theory and to the specialist as a source of reference.

J. M. MARStrand

\section{Structure and Reaction}

Chemical and Mechanical Behaviour of Inorganic Materials. By Alan W. Searcy, David V. Ragone and Umberto Colombo. (Academia Nazionale dei Lincei, Eleventh Course of the Guido Donegani Foundation, Tremezzo (Lake Como), Italy, September 8-20, 1968.) Pp. xxiv +715 . (Wiley-Interscience: New York and London, December 1970.) $£ 13.00$.

THIs large book contains the lectures presented to a summer school arranged "for graduates in chemistry and physics who have minimal backgrounds in materials science", people who are assumed to know little of crystal defects or of solid-state chemical reactions. To the materials scientist's jaded palate it is a refreshing, because unusual, diet; 29 chapters deal first with general chemical and defect principles, and subsequently with particular applications of some of these principles. The early chapters lean heavily on thermodynamic interpretations: thus one of Searcy's five chapters illuminatingly interprets the thermo- dynamics of reactions involving solids and gases largely in terms of the entropy of gaseous products, and this is developed in de Maria's chapter on unfamiliar vapour molecules. The statistical mechanics of evaporation/condensation equilibria and the stability of various refractories are analysed in two complementary chapters.

The book moves on to structural aspects of solids. Heavy emphasis is placed in two chapters by Ragone on the physical chemistry of defects in solids and on their relation to the extent of nonstoichiometry. A particularly illuminating chapter later in the book is that by Reiss on the "electron as a chemical entity in solids". In effect, the electron is treated as a defect in equilibrium with donors and acceptors, and the origin of solute/solute solubility cross-effects in semiconductors is very clearly set out on mass-action principles. Reiss's chapter, which is frankly didactic in a field where many authors strive only to be encyclopaedic, is of a special quality: one is reminded of Hinshelwood's style in his Structure of Physical Chemistry.

Other chapters-more familiar to a materials scientist-deal with diffusion (Ascoli) and, very comprehensively, with dislocation geometry and dynamics and the special behaviour of dislocations in controlling ductility and fracture in ceramics (Conrad). Kelly surveys fibrecomposites. Various aspects of surfaces are considered in several chapters. Ragone is interesting on topics such as the distinction between surface tension and energy, but somewhat short on practical implications of surface energy, such as thermal etching. Chemical surface (gas/solid) reactions also receive due attention.

The last part of the book deals with particular classes of materials. Zarzycki elegantly surveys the basic features that characterize the structure of oxide glasses, Bokros surveys the world of pyrolytic carbon which is almost his own creation, and two chapters deal with the very special problems of nuclear fuels. Another chapter surveys a number of special materials such as glass-ceramics and ferroelectrics (really too short to be very useful), and another provides extensive insight into ceramic-based batteries. This chapter is scientifically and technologically particularly interesting. The rear is brought up by Frank, who writes on the Earth as a ceramic body. $\mathrm{He}$ writes with all his familiar verve and éclat; it is most instructive to observe how many qualitative deductions about the nature of heat transport and tectonic processes in the Earth's core, mantle and crust can be drawn with the help of cunning order-of-magnitude calculations. A book much concerned with the manmade thus finishes with a deep look at levels inaccessible to man.

$$
\text { R. W. CAHN }
$$

